\title{
Cultural scripts guide recall of intensely positive life events
}

\author{
Katherine A. Collins \\ Wellesley College, Wellesley, Massachusetts \\ AND
}

David B. Pillemer, Zorana Ivcevic, And Rachel A. Gooze

University of New Hampshire, Durham, New Hampshire

\begin{abstract}
In four studies, we examined the temporal distribution of positive and negative memories of momentous life events. College students and middle-aged adults reported events occurring from the ages of 8 to 18 years in which they had felt especially good or especially bad about themselves. Distributions of positive memories showed a marked peak at ages 17 and 18. In contrast, distributions of negative memories were relatively flat. These patterns were consistent for males and females and for younger and older adults. Content analyses indicated that a substantial proportion of positive memories from late adolescence described culturally prescribed landmark events surrounding the major life transition from high school to college. When the participants were asked for recollections from life periods that lack obvious age-linked milestone events, age distributions of positive and negative memories were similar. The results support and extend Berntsen and Rubin's (2004) conclusion that cultural expectations, or life scripts, organize recall of positive, but not negative, events.
\end{abstract}

Three decades ago, most empirical research on memory was conducted under carefully controlled experimental conditions. The laboratory approach has produced highly replicable and broadly applicable findings, such as primacy and recency effects in short-term memory and timedependent decay in long-term memory. In recent years, psychologists have examined memory by using increasingly diverse methodologies. One prominent contemporary approach is to examine memories of personal events experienced in the context of everyday activities, rather than in cognitive psychology laboratories (e.g., Neisser, 1982; Pillemer, 1998; Rubin, 1986, 1996). Despite a lack of experimental control over encoding conditions and memory content, studies in which everyday memory has been examined also have generated robust findings. In particular, several investigators have examined age distributions of personal memories over the life span and have identified striking temporal regularities.

One robust temporal pattern involves what has come to be known as the reminiscence bump. Rubin and colleagues have used a variety of methodologies to elicit personal memories from middle-aged and older adults, and they consistently have found a disproportionate number of remembered events that occurred between the ages of 10 and 30 years (e.g., Rubin, Rahhal, \& Poon, 1998). Memory incidence typically rises sharply during childhood and adolescence, peaks in late adolescence or early adulthood, and then declines. This reminiscence bump is apparent across cultures, including the United States,
England, Japan, China, and Bangladesh (Conway, Wang, Hanyu, \& Haque, 2005). Berntsen and Rubin (2002) summarized the pattern of results as follows: "For people over the age of 40, information encoded during adolescence and early adulthood is remembered better than information encountered in the surrounding periods of life" (p. 637). A number of theoretical explanations have been offered for the bump, including the novel, transitional, and memorable nature of events occurring during adolescence and early adulthood, the rapid development of personal identity and a coherent autobiographical life story at this time, and cultural expectations, or life scripts, that identify ages $15-30$ as the period within which landmark personal events are most likely to occur (Berntsen \& Rubin, 2002; Conway \& Pleydell-Pearce, 2000; Rubin et al., 1998).

Recent research has provided a basis for evaluating these alternative explanations. An elegant series of studies by Berntsen and Rubin provides strong support for culturally shared life scripts as an organizing force contributing to the reminiscence bump. Berntsen and Rubin (2002) included memory questions in a large-scale Danish interview study. Adult participants were asked to remember an extremely happy, sad, important, or traumatic memory and to report their age at the time of the event. Age distributions differed strikingly as a function of emotional valence. For participants over age 40, the happiest and most important events showed the expected reminiscence bump, with a peak in the 20 s, whereas the saddest

D. B. Pillemer, david.pillemer@unh.edu 
and most traumatic memories did not. In a second Danish interview study, Rubin and Berntsen's (2003) memory probes included events representing extreme fear, pride, jealousy, love, anger, and importance. Again, the distributions of the positive memories demonstrated the reminiscence bump much more clearly and consistently than did those of the negative memories.

Rubin and Berntsen (2003; Berntsen \& Rubin, 2002) argued that the divergent pattern of findings for positive and negative memories supported a life script theoretical explanation. Landmark positive events are a prominent component of cultural expectations: "The retrieval of autobiographical memories is governed by culturally shared representations of the prototypical life cycle that locate the majority of important transitional events in young adulthood and favor positive events" (Rubin \& Berntsen, 2003 , p. 2). These positive events may include high school graduation, going to college, getting a first job, and marriage. When probed to report a positive memory, cultural expectations may guide the retrieval search to late adolescence and early adulthood, where these scripted events typically occur. In contrast, life scripts are less likely to specify particular age ranges for major negative events. Negative emotions may be strongly felt when expectations go awry, but the timing of these feelings is not part of the prototypical life script.

In a follow-up study, Rubin and Berntsen (2003) examined life scripts more directly. College students in Denmark completed a questionnaire in which they were asked to "imagine an average 70 year old, who is looking back at his or her life," and to "give an estimate of this person's age at the time when each of these events took place" (p. 9). The event prompts were the same as those given to the participants in Rubin and Berntsen's earlier memory studies: extremely happy, sad, important, traumatic, angry, in love, jealous, proud, and afraid. College students' expectations for the timing of extreme events were fairly consistent with the actual age distributions of memories reported by older adults. For example, students' expectations for the age at which an elderly individual would locate an extremely happy memory produced a sharp bump that peaked in early adulthood, whereas their age expectations for extremely sad events were relatively flat, showing only a gradual increase over the life span.

More recently, Berntsen and Rubin (2004) asked Danish adults to imagine a 100-year-old who is reflecting on his or her life and to estimate the person's age when several different types of events occurred: extremely happy, extremely sad, extremely important, most traumatic, extremely in love, and extremely afraid. As in previous studies, age-related distributions for positive events showed a sharp rise in late adolescence and a peak in the 20s, whereas the distributions of negative events were relatively flat. The researchers also asked Danish undergraduates to list the seven most important events that are likely to occur in a typical person's life and to indicate the expected ages of occurrence. Again, the age distribution of positive, but not negative, expected events showed the predictable reminiscence bump.
Like Rubin and Berntsen (2003), we elicited intensely positive and negative personal memories. College students (Studies 1 and 2) were asked to report memories of events occurring from the ages of 8 to 18 , an interval that is part of the sharply upward sloping component of the reminiscence bump found in previous studies. Comparing age distributions of positive and negative memories provided a further test of the cultural script hypothesis: Finding a marked peak in late adolescence for positive memories and a relatively flat distribution for negative memories would concur with the results of previous research and would support the cultural script explanation for the reminiscence bump.

Berntsen and Rubin's (2002) analysis of the happiest and saddest memories reported by Danish adults most closely parallels the analysis in the present study. For participants in their 20 s, who, like our respondents, had not yet passed through the years marking the reminiscence bump, happiest memories show a steep age-related increase up to age 20 (as did proudest memories; Rubin \& Berntsen, 2003), whereas the increase for saddest memories is more tempered and begins to flatten before age 20 . Berntsen and Rubin's data presentation does not allow for a precise, year-by-year comparison of positive and negative memory distributions. In contrast, our analyses permit detailed year-by-year age comparisons.

The participants in Rubin and Berntsen's (2003) studies reported the ages corresponding to their highly emotional memories, but they were not asked to describe the remembered episodes. As a result, it was not possible to test the cultural script hypothesis directly, by examining memory content. In contrast, the participants in the present studies wrote out their salient memories of childhood and adolescence. Memories that are consistent with cultural scripts will share certain qualities: Remembered events should be experienced by a substantial number of people in the culture; the events' importance within the life course should be part of shared cultural knowledge; and the events should be linked to a specific age or a limited age range. If positive memories are overrepresented in late adolescence because retrieval is guided by life scripts, event descriptions should focus frequently on common and predictable cultural milestones. One prominent source of positive memories for middle class residents of the U.S. may be events surrounding the major life transition from high school to college, such as high school graduation and college acceptance. In contrast, the content of negative memories should show no such consistent pattern.

As a further test of the cultural script hypothesis, college students in Study 3 provided memories from ages 10 to 15 , a life period that does not contain obvious age-linked positive landmark events or transitions. In the absence of prominent developmental milestones, temporal distributions of positive and negative memories should be similar and should show only a gradual age-related decay (Berntsen \& Rubin, 2002). In Study 4, middle-aged college graduates recounted events from a life period marked by notable transitional events (ages 
8-18) and also from a life period following the reminiscence bump, for which culturally prescribed, age-linked landmarks are less prominent (ages 34-44). We expected temporal distributions of positive and negative memories, and the corresponding incidence of age-linked cultural milestones, to diverge for the $8-18$ age interval, but not for the 34-44 age interval.

\section{STUDY 1}

\section{Method}

Participants. The participants included 92 students (mean age $=$ 20.73 years) attending Wellesley College, a liberal arts college for women. With respect to ethnicity, $72.8 \%$ of the participants selfidentified as Caucasian, $12 \%$ as Asian, $6.5 \%$ as Black or African American, $3.3 \%$ as Hispanic, and $5.5 \%$ as mixed. The participants tended to come from well-educated families: $84.7 \%$ of their mothers and $80.4 \%$ of their fathers were college graduates.

Questionnaire and Procedure. Each participant completed a paper-and-pencil questionnaire. The first section asked the participants to provide their age, gender, and ethnic background, to indicate the highest level of education attained by each of their parents, and to describe their own educational history. The second section consisted of Rosenberg's (1965) global self-esteem scale. Results using this scale were not relevant to the present study and will not be reported here.

The third section consisted of three memory prompts and a number of follow-up questions for each memory elicited. The first two memory prompts directed the participants to describe in detail a specific memory of one time when they had felt "especially good" about themselves between the ages of 8 and 18 and to describe in detail a specific memory of one time when they had felt "especially bad" about themselves between the ages of 8 and 18. To control for order effects, half of the participants (alternating according to their identification codes) gave a positive memory first, and half gave a negative memory first. The third prompt asked the participants to provide an additional memory involving an incident that precipitated either "especially good" or "especially bad" feelings about the self; the choice of either a positive or a negative memory was left to the respondents. Responses to the third memory prompt will not be reported here.

Following each memory prompt, the participants estimated their age in years at the time of the remembered experience; indicated on a 4-point scale how often they had discussed the memory with others; rated on 5-point scales the intensity of emotions felt both at the time of the event and currently; indicated the quality of emotions felt at the time and currently (positive, negative, mixed, or neutral); rated on a 5-point scale the impact of the event on current feelings about the self as a person; and rated on a 5-point scale how difficult it was to retrieve a memory in response to the prompt.

Groups of participants were tested in quiet spaces on campus. The participants were initially presented with a brief written introduction explaining their right to confidentiality and to discontinue their participation at any time. Once they had read the introduction, they signed a voluntary consent form and were allotted as much time as necessary to complete the questionnaire. When finished, they received a debriefing form and $\$ 5$ payment.

\section{Results}

Frequency distributions of memories reported at each age in response to positive and negative memory prompts are presented in Figure 1; these distributions were markedly different. Almost one half (46.7\%) of the positive memories were assigned to age 17 or 18 . In contrast, the negative memory distribution is relatively flat, and only $18.5 \%$ of remembered events occurred at age 17 or 18 .
The mean age at which the participants remembered feeling especially good about themselves was significantly higher $(M=15.46$ years, $S D=2.54)$ than the age at which the participants remembered feeling especially bad about themselves $(M=13.37$ years, $S D=3.01)[t(91)=$ $5.29, p<.001]$. A nonparametric test (Wilcoxon signed ranks test) produced a similar finding for this and for subsequent statistical tests involving the age of memories.

The participants' ratings of positive and negative memories were compared. Ratings of original emotional responses to experiences in which they felt especially bad about themselves $(M=4.20, S D=0.85)$ were stronger than the ratings of emotional responses to events in which they felt especially good about themselves $(M=3.64$, $S D=1.02)[t(91)=-4.44, p<.001]$. However, the participants indicated that they had discussed their positive memories $(M=2.27, S D=0.92)$ more often than their negative memories $(M=2.00, S D=0.93)[t(91)=2.33$, $p=.022]$ and that positive memories had a greater impact on how they currently felt about themselves $(M=2.71$, $S D=1.08)$ than did negative memories $(M=2.18, S D=$ 1.09) $[t(91)=3.65, p<.001]$.

A life script explanation for the elevated incidence of positive memories during late adolescence would predict that a substantial proportion of positive memories represent culturally prescribed landmark events. To explore this idea, we analyzed the content of memories corresponding to the ages of 17 and 18, at just the point at which the incidence of positive memories, but not negative memories, increases dramatically. We looked for clusters of similar events that fulfilled certain conditions: (1) They are experienced by substantial numbers of people, (2) they are defined as important or significant by the culture, and (3) they are linked directly to a particular age or a narrow age range. An initial reading of the positive memory

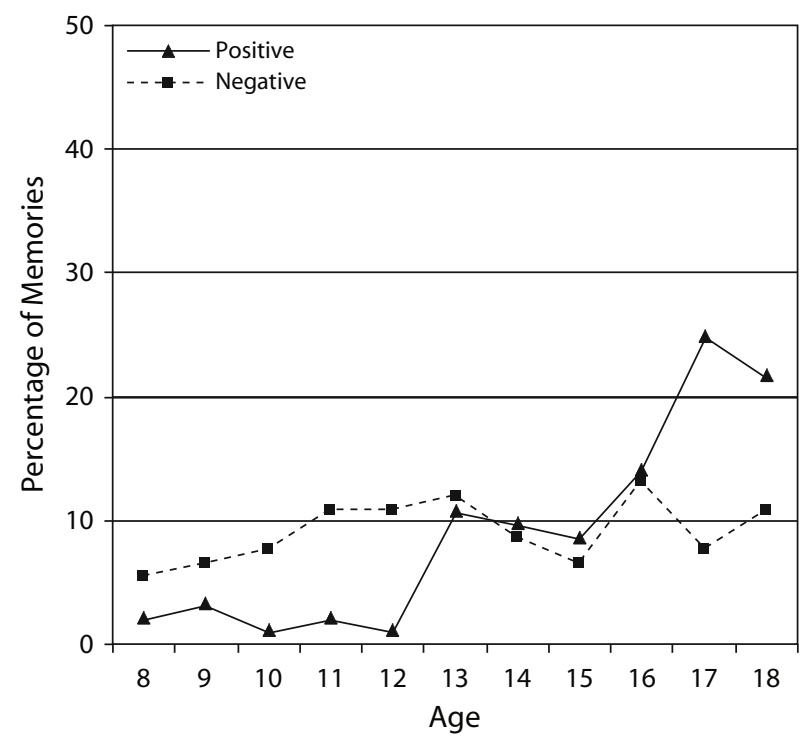

Figure 1. Age distributions of positive and negative memories between the ages of 8 and 18 years for Wellesley College women (Study 1). 
narratives identified several events that are part of the cultural script for the late-adolescent life transition from high school to college: college acceptance, high school graduation, awards received during the senior year of high school or at graduation, and formal senior social events (such as proms or homecoming). Two researchers read all the memories of events that had occurred at age 17 or 18 and jointly assigned them to the content categories listed above. Agreement with a third independent coder as to whether a landmark event was present or absent in the memories was $94.9 \%$; disagreements were resolved through discussion.

One third (14/42) of the positive memories from the ages of 17 and 18 represented events connected to the high school to college transition, including college acceptance $(11.9 \%)$, senior awards (11.9\%), high school graduation (7.1\%), and senior social events (2.4\%). These transitional events, with the possible exception of senior awards, are almost universally experienced by students who go on to attend highly selective colleges. Award ceremonies are predictable components of high school graduations and would be salient for many of our high-achieving participants.

Predictably, content analyses of the small number $(n=$ 17) of negative memories from ages 17 and 18 failed to identify distinctive clusters of culturally shared landmarks. Although the remembered events were personal milestones (e.g., receiving bad grades in school or relationship problems or breakups), they did not conform to a cultural script tied to a particular age or developmental transition. The only negative landmark events were two instances of failure to attain a positive milestone (college rejection).

\section{STUDY 2}

The Study 1 participants were female and attended the same elite college. Study 2 was conducted to determine whether the consistent pattern of results obtained in Study 1 would replicate in a different educational setting and with men as well as women.

\section{Method}

Participants. The participants included 43 women and 51 men (mean age $=20.3$ years) attending Babson College. The participants were enlisted through announcements in large sophomore seminars or were recruited with advertisements posted in the student center With respect to ethnicity, $79.8 \%$ of the participants self-identified as Caucasian, $7.4 \%$ as Asian, $5.3 \%$ as Hispanic, $1.1 \%$ as Black or African American, and 6.5\% as mixed/other. With respect to family education, $71.3 \%$ of their mothers and $71.3 \%$ of their fathers were college graduates.

Questionnaire and Procedure. The test materials and procedures employed in Study 2 were identical to those utilized in Study 1.

\section{Results}

Age distributions for positive and negative memories are presented in Figure 2. When asked to recall a time at which they had felt especially good about themselves between the ages of 8 and 18, most of the participants (70.3\%) recounted events that had occurred at the age of 17 or 18 . When asked to report a memory of a time when they had felt especially bad about themselves, the age increase was much flatter: Only $34.0 \%$ of the participants described incidents that had occurred at the age of 17 or 18. Positive memories represented events that had occurred significantly later in adolescence ( $M=16.36$ years, $S D=2.37)$ than had those represented by negative memories $(M=$ 14.51 years, $S D=2.99)[t(93)=5.28, p<.001]$.

As in Study 1, the respondents' ratings of their emotional responses to negative events $(M=4.24, S D=0.88)$ were higher than their ratings of emotional responses to positive events $(M=3.73, S D=1.07)[t(93)=-4.22$, $p<.001]$. However, positive memories had greater current emotional salience (positive, $M=2.82, S D=0.98$; negative, $M=2.44, S D=1.13$ ) $[t(93)=2.94, p=.004]$. Moreover, the participants reported experiencing greater ease when thinking of a positive $(M=1.68, S D=0.89)$ than when thinking of a negative $(M=2.22, S D=1.14)$ memory $[t(93)=-3.44, p=.001]$ and reported discussing their positive memories $(M=2.67, S D=0.90)$ more often than their negative memories $(M=2.36, S D=0.84)$ $[t(93)=2.59, p=.011]$. Positive memories $(M=3.14$, $S D=0.93)$ had a greater impact on how the participants currently felt about themselves than did negative memories $(M=2.42, S D=1.16)[t(93)=5.22, p<.001]$.

Age distributions for positive and negative memories were constructed for men and women separately; the separate distributions were consistent with the pattern observed in the total sample. When asked to recall a time at which they had felt especially good about themselves, a majority of the men $(70.6 \%)$ and women $(69.8 \%)$ recounted events that had occurred at the age of 17 or 18 . In contrast, only about one third of the negative memories reported by the men $(31.3 \%)$ and by the women $(37.2 \%)$ had occurred at ages 17 or 18 . The men's and women's mean positive memory age (female, $M=16.44, S D=$ 2.15; male, $M=16.29, S D=2.56$ ) and negative memory

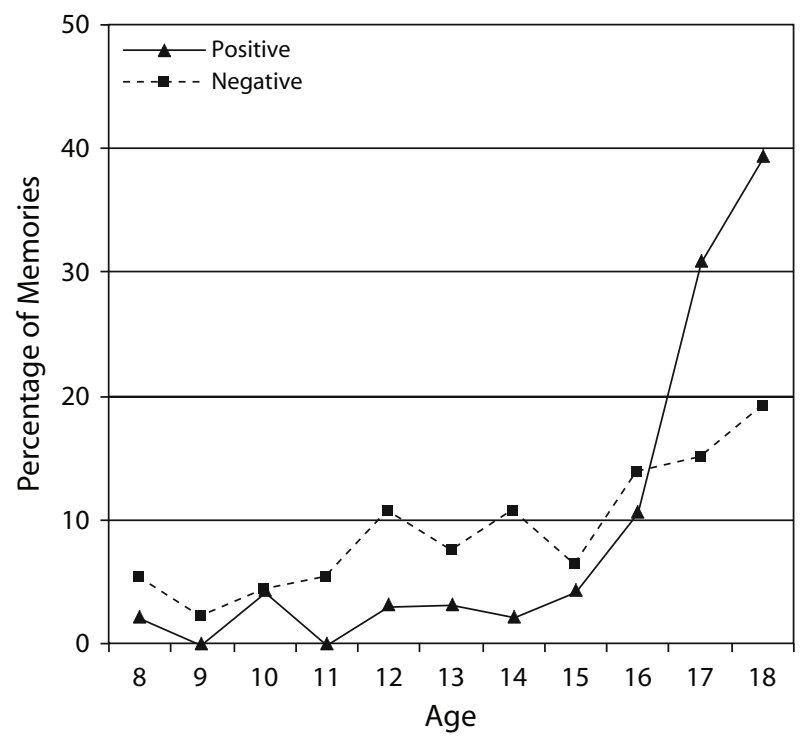

Figure 2. Age distributions of positive and negative memories between the ages of 8 and 18 years for Babson College students (Study 2). 
age (female, $M=14.81, S D=2.72$; male, $M=14.25$, $S D=3.21$ ) were similar; gender differences did not approach statistical significance.

As in Study 1, the life script explanation for the sharply increasing incidence of positive memories in late adolescence was evaluated by examining the content of memories from the ages of 17 and 18, the point at which the positive and negative memory distributions diverge. Two researchers read all the memories and jointly assigned them to the content categories used in Study 1. Agreement with an independent third coder as to the presence or absence of landmark events in the memories was $95.9 \%$; disagreements were resolved by discussion.

Over one third (26/66) of positive memories described a scripted event accompanying the major life transition from high school to college: college acceptance (15.2\%), senior awards (16.7\%), high school graduation $(4.5 \%)$, and formal senior social events $(3.0 \%)$. In contrast, content analyses of the 32 negative memories did not identify clusters of age-linked scripted events. The memories frequently focused on relationship breakups or problems, but these issues were not tied consistently to particular ages or developmental transitions (such as breaking up with a high school sweetheart in anticipation of leaving for college). Another cluster of negative memories reported by the males focused on problems with the law (e.g., speeding tickets or arrest for harassment), but these did not conform to a well-defined age-linked script (such as underage driving).

\section{STUDY 3}

The participants in Studies 1 and 2 provided memories of events occurring within a transitional lifetime period marked by notable landmark events. Although content analyses are consistent with a life script explanation for the age-related increase in positive, but not negative, memories, an alternative interpretation also is plausible. When asked to provide positive and negative self-esteem memories that occurred within a circumscribed life period, people could show a general tendency to report a greater number of recently occurring positive events than negative events. Ross and Wilson (2002, 2003; Wilson \& Ross, 2001, 2003) identified a "self-enhancement function of autobiographical memory" (Wilson \& Ross, 2003, p. 139). People are motivated to view current selves in a positive light, and they "regard negative outcomes as more distant than positive episodes" (p. 143). In the present studies, the request for memories that represented highly emotional self-evaluations could activate this self-enhancing quality of autobiographical memory. If such a general self-enhancement function is operative, positive memories may show a greater recency effect than do negative memories, independently of the specific age boundaries specified in the memory probe.

To evaluate this possibility, we asked college students to provide memories occurring between the ages of 10 and 15-a life period embedded in the 8-18 age interval used in Studies 1 and 2, but one that did not include the prominent age-linked transition from high school to college. Ac- cording to the life script hypothesis, memory distributions of positive and negative memories should be similar when such milestones are missing.

\section{Method}

Participants. The participants included 99 women and 54 men (mean age $=18.88$ years) who were enrolled in psychology courses at the University of New Hampshire and who received course credit for their participation. With respect to ethnicity, $92.2 \%$ selfidentified as Caucasian, 2.6\% as Black or African American, 2.0\% as Asian, $2.0 \%$ as Hispanic, and $1.3 \%$ as mixed race/other. With respect to family education, $58.2 \%$ of their mothers and $54.3 \%$ of their fathers were college graduates.

Questionnaire and Procedure. The questionnaire and procedures were similar to those employed in Studies 1 and 2, except that the memory prompts targeted different life periods. The first two memory prompts asked the participants to describe in detail their earliest childhood memory of one time when they had felt especially good about themselves and one time when they had felt especially bad about themselves. The second two prompts asked for a specific memory of one time when they had felt especially good about themselves between the ages of 10 and 15 and one time when they had felt especially bad about themselves between the ages of 10 and 15 . The order of positive and negative memories was counterbalanced. Only memories from the 10-15 age interval are relevant to the theoretical issues addressed in this article.

\section{Results}

Frequency distributions of memories reported at each age in response to positive and negative memory prompts are presented in Figure 3. In contrast to the results of Studies 1 and 2, and consistent with predictions based on the absence of culturally prescribed landmarks (Berntsen \& Rubin, 2002), the distributions are very similar, each showing only a modest age-related decay. The mean ages at which the respondents remembered feeling especially good about themselves $(M=13.05, S D=1.63)$ and feeling especially

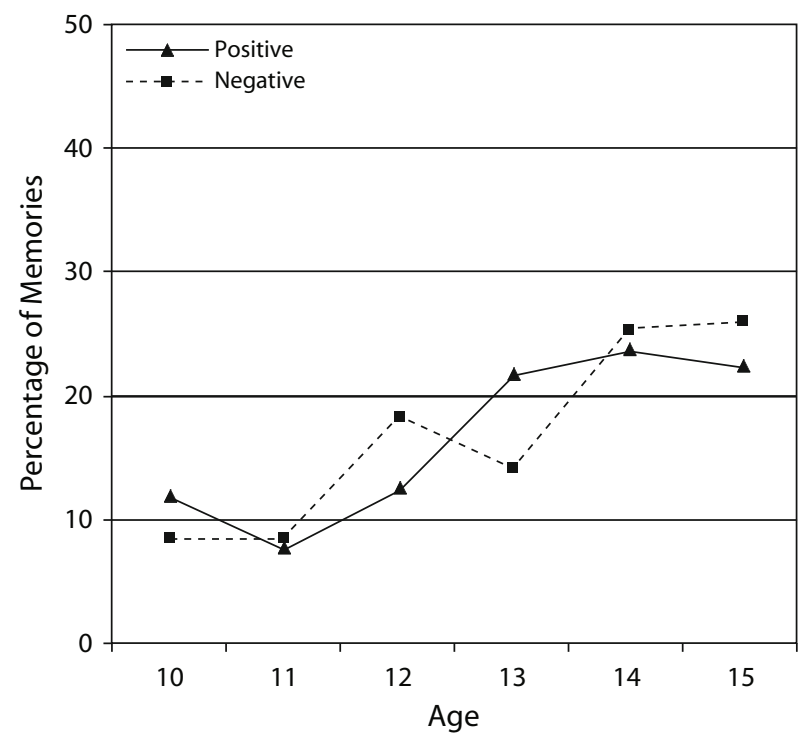

Figure 3. Age distributions of positive and negative memories between the ages of 10 and 15 years for University of New Hampshire students (Study 3). 
bad about themselves $(M=13.17, S D=1.60)$ were not significantly different $[t(142)=-0.71, p=.481]$.

\section{STUDY 4}

To establish a more direct connection between our findings and those in studies of the reminiscence bump, which typically have employed older samples, we replicated our initial studies using middle-aged Wellesley alumnae as respondents. When asked to report memories of events from the age of 8 to 18 that had made them feel especially good or bad about themselves, we expected the older participants to produce the same pattern of results as that found for college students: The positive memory distribution should peak in late adolescence, whereas the negative memory distribution should be flatter. Content analyses of older respondents' memories should identify substantial numbers of scripted and predictable positive events, but not negative events, occurring at the ages of 17 and 18 .

Conducting studies with older adults also permitted a second test of the alternative explanation that people may show a general tendency to report a greater number of recent positive self-evaluative episodes than negative ones, regardless of the presence or absence of age-linked landmark events that normally occur within the specified recall interval. The participants reported memories either from a life period commonly populated by scripted life events (ages 8-18), or from a life period following the reminiscence bump for which age-related milestones were less prominent (ages 34-44).

\section{Method}

Participants. The participants were alumnae of the Wellesley graduating classes of 1978 and 1979. The alumnae were approximately 2 years older than the upper limit of the age 34-44 recall interval, just as the college students in Studies 1 and 2 were approximately 2 years older than the upper limit of the age 8-18 recall interval. In a first mailing, questionnaires were sent to a random sample of 248 members of the class of 1978. Because the response rate was low (20 completed questionnaires were received), questionnaires were sent to 280 additional members of the class of 1978 and to 513 members of the class of 1979 . The samples included all the alumnae for whom the Wellesley College Alumnae Association had current contact information. For the first mailing, the respondents returned both the questionnaire (containing highly emotional personal information) and a signed consent form in the same envelope. In an attempt to increase the return rate, for the follow-up mailings, the consent forms and questionnaires were returned separately. A substantial number of questionnaires could not be delivered because addresses were no longer current. In addition, several participants from the class of 1978 were omitted because they returned one of a small subset of questionnaires that contained a typographical error.

The final sample consisted of 115 alumnae, 53 from the class of 1978 and 62 from the class of 1979; this represented an overall participation rate of $11 \%$. The average age of the participants was 46.8 for the class of 1978 and 45.8 for the class of 1979 . Because Wellesley is a women's college, all the participants were female. With respect to ethnicity, $78.3 \%$ of the participants self-identified as Caucasian, 7.8\% as Asian, 6.1\% as Black or African American, and $7.9 \%$ as mixed/other.

Questionnaire and Procedure. The participants completed a questionnaire that they received by mail. A cover letter briefly described the study, indicated that participation was voluntary, and stated that responses would be kept confidential. The respondents returned the questionnaire and a voluntary consent form in an enclosed, stamped envelope. The participants were told that they would receive a summary of the results after the study had been completed.

On the memory questionnaire, the participants first provided their age and ethnic background. Then the participants described four specific memories. They described two memories from ages $8-18$ and two memories from ages $34-44$. For each age period, they described one event when they had felt especially good about themselves and one event when they had felt especially bad about themselves. The participants were assigned sequentially to one of eight possible memory orders.

\section{Results}

Frequency distributions of positive and negative memories corresponding to the 8-18 age interval are presented in Figure 4. Substantially more positive (39.5\%) than negative $(21.0 \%)$ memories were assigned to ages 17 and 18 . The mean age at which the participants remembered feeling especially good about themselves was significantly older $(M=14.80, S D=2.82)$ than the mean age at which the participants remembered feeling especially bad about themselves $(M=13.89, S D=2.96)[t(113)=2.33, p=$ .022].

The life script explanation for the differing distributions of positive and negative memories was evaluated by conducting content analyses of remembered events from the ages of 17 and 18. Two coders read all the memories and jointly assigned them to the personal landmark categories used in Studies 1 and 2. Agreement with an independent third coder as to the presence or absence of landmark events was $94.3 \%$; disagreements were resolved through discussion. Almost one half (22/46) of the positive memories described a transitional milestone: college acceptance $(32.6 \%)$, a senior year award (13.0\%), and

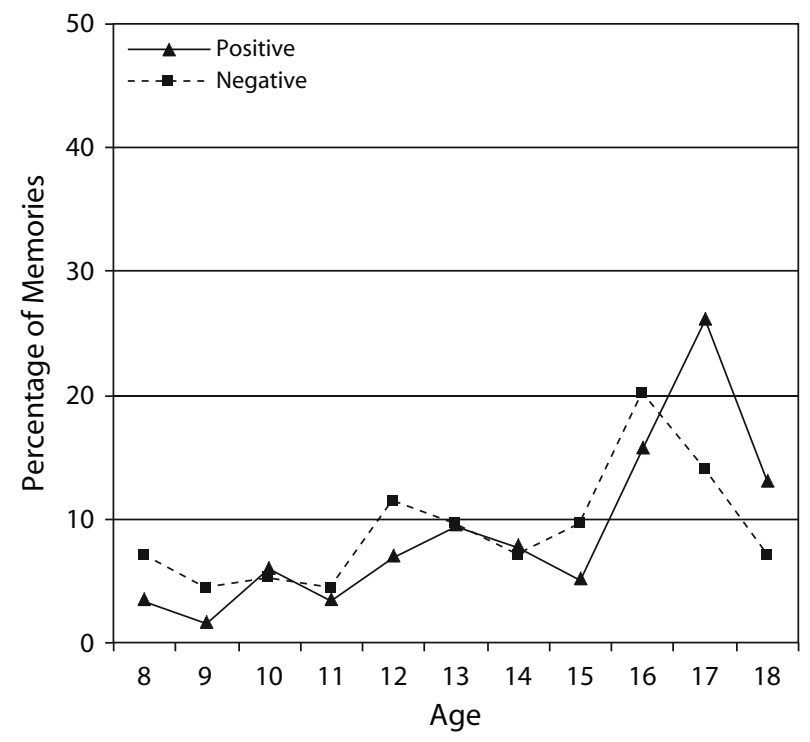

Figure 4. Age distributions of positive and negative memories between the ages of 8 and 18 years for Wellesley College alumnae (Study 4). 
high school graduation (2.2\%). In contrast, only 2 of the 24 negative memories focused on one of these predictable events: college rejection.

Frequency distributions of positive and negative memories corresponding to the 34-44 age interval are presented in Figure 5. The mean age at which the participants remembered feeling especially good about themselves $(M=$ $39.68, S D=3.15$ ) was not significantly different from the mean age at which the participants remembered feeling especially bad about themselves $(M=39.29, S D=3.12)$ $[t(101)=0.91, p=.364]$.

Memory distributions from ages 34-44 showed modest peaks at ages 40 (positive memories) and 42 (negative memories). A cluster of positive memories (6 out of 22 memories) from age 40 described events surrounding the landmark 40th birthday. The negative memories from age 42 involved salient personal events (e.g., problems related to work or family) that were not tied to age 42 in any scripted or obvious way.

\section{GENERAL DISCUSSION}

Participants in three studies were asked to describe personal memories from ages $8-18$ in which they had felt especially good or bad about themselves. The pattern of results is highly consistent. Positive moments of selfevaluation are overrepresented in the late teen years, at ages 17 and 18. In contrast, age distributions of negative episodes are relatively flat. These temporal patterns are evident for college students and middle-aged adults and for men and women.

Our findings confirm and extend previous research on the reminiscence bump. Berntsen and Rubin (2002; Rubin $\&$ Berntsen, 2003) asked adults to remember extremely positive and negative events that had occurred at any time in their pasts and to estimate the corresponding dates of occurrence. They discovered that the incidence of positive, but not negative, events rises sharply during adolescence, with a peak in the 20 s. In the present studies, memory incidence also increases markedly in late adolescence for positive, but not negative, episodes. Berntsen and Rubin (2002) proposed that temporal distributions of positive and negative memories differ because cultural expectations or life scripts direct the memory search to positive (but not negative) milestones predictably located in adolescence and early adulthood. Whereas sharply negative events are deviant and do not fit a culturally prescribed timetable, "most important and positive transitional events are supposed to take place between 15 and 30" (Berntsen \& Rubin, 2002, p. 640).

Because the participants in previous studies of the reminiscence bump did not provide written memory narratives, the authors were unable to evaluate the life script hypothesis directly. In the present studies, the participants wrote out detailed memories that were examined for the presence of expected cultural landmarks. In all the studies, a substantial number of positive episodes occurring in late adolescence involved the salient and significant life transition from high school to college, including high school graduation and awards and college acceptance. Negative

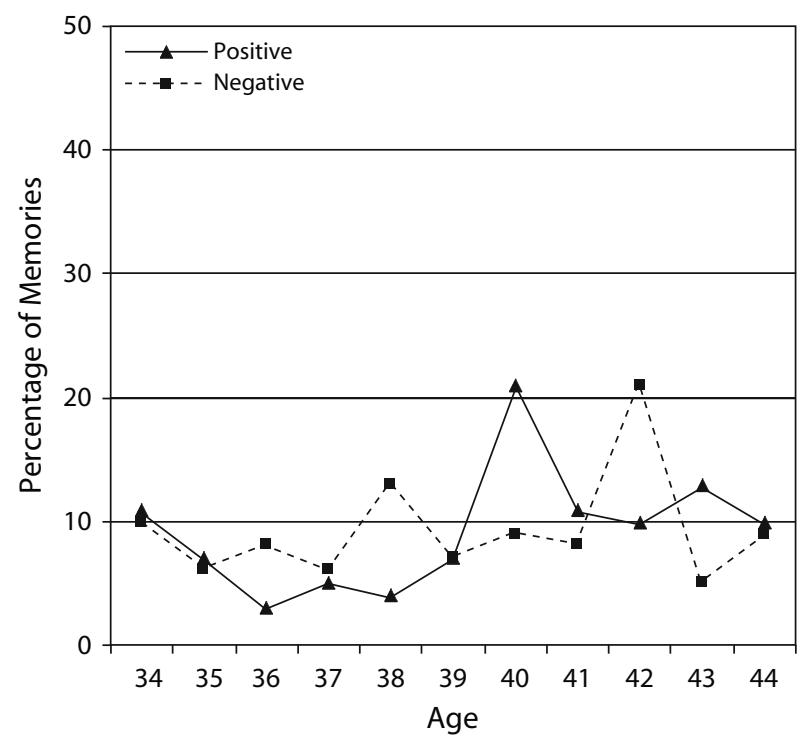

Figure 5. Age distributions of positive and negative memories between the ages of 34 and 44 years for Wellesley College alumnae (Study 4).

memories of adolescence portrayed a variety of personal landmark events, but they did not reveal well-defined, age-linked negative cultural scripts. For example, memories of romantic relationship breakups were not linked consistently to specific ages or developmental transitions, such as leaving for college.

Cultural milestones make up some, but not all, positive memories occurring at ages 17 and 18. Shared expectations about the importance of the high school to college transition may direct the positive memory search to this targeted age period, rather than to particular events. Respondents then choose among memories located within the transitional period. Some participants may select positive memories other than emblematic stereotypical landmarks. For example, substantial numbers of positive memories from the ages of 17 and 18 involved various types and levels of success or recognition in sports, academics, or the arts, but these episodes were not tied explicitly to the scripted transition from the senior year in high school to the first year in college.

Additional support for the life script hypothesis comes from analyses of college students' ratings of behaviors and beliefs associated with their reported memories. Positive memories were discussed with others more frequently than were negative memories, positive memories were easier to recall, and positive memories were rated as having a greater impact on current feelings about the self. These findings are consistent with the idea that extremely positive memories of the transition from high school to college represent well-rehearsed, socially shared events that fit predictably and prominently in the prototypical life story. Positive memories of adolescent achievement landmarks may retain their accessibility because of their "enduring association with the current goals of the working self" (Conway \& Pleydell-Pearce, 2000, p. 280). 
By including memory prompts that targeted life periods other than late adolescence and early adulthood, we were able to rule out an alternative explanation for the differing age distributions of positive and negative memories. It is possible that positive, but not negative, memories cluster at the older end of the 8-18 age range because of the self-enhancing quality of autobiographical memory (Wilson \& Ross, 2003). The participants may have enhanced their current conceptions of self by recalling positive selfevaluative episodes that had occurred recently and by distancing themselves from negative self-evaluative moments. The college students in Study 3 recounted memories from ages 10-15, and the middle-aged adults in Study 4 recounted memories from ages $34-44$. In these instances, remembered positive events were not more recent than negative events. Had a general self-enhancing function of autobiographical memory been operative, it also should have been evident in the temporal distribution of memories corresponding to these recall intervals, which did not include obvious age-linked cultural milestones. In line with the life script account, the modest positive memory bump at age 40 reflects a cluster of events marking the pivotal 40th birthday. No such explanation is apparent for the modest negative memory bump at age 42 .

A substantial proportion of positive memories from ages 17 and 18 focused on getting into college. College acceptance illustrates the potential directive influence of cultural expectations on memory. Tekcan (2001) asked college students to recall the precise circumstances in which they learned of their acceptance to college. Most participants were able to recall personal details of hearing the news. Mean emotion ratings were very strong, and the participants reported telling other people the story of how they had heard the news an average of approximately five times. Popular books, with titles such as Getting In: Inside the College Admissions Process (Paul, 1995), Making it Into a Top College (Greene \& Greene, 2000), and The College Admissions Mystique (Mayher, 1998), reflect the "current fascination middle-class American culture has with selective college admissions" (Mayher, 1998, p. 13). Descriptions of the admissions process invariably contain a section on "getting the news," the eagerly anticipated April announcement date of acceptances and rejections: "This is the moment you have been waiting for. No matter how much perspective any of us bring to such an event, it is hard to approach judgment day without a lump in the throat" (Mayher, 1998, p. 65).

It is little wonder that enduring memories of positive self-worth so often center on this singular moment. According to Berntsen and Rubin (2004), such events "come with a consequentiality and importance that is culturally agreed upon and are therefore likely to be better encoded than events that do not" (p. 439). In addition, memories of personal landmarks are frequently thought and talked about, and they continue to anchor enduring beliefs about the self (Pillemer, 1998); our participants reported talking more frequently about positive than about negative events. Memories of college rejections presumably are less salient because they are less likely to be closely tied to current self-conceptions, which focus on the college actually attended, rather than on missed opportunities from the past.

The positive events marking late adolescence focused on educational experiences and achievement. The participants were students or graduates of competitive colleges, and as such, achievement milestones may hold special and enduring significance. Future research should include individuals who did not attend college. One possibility is that late adolescence will not provide as intense and significant a developmental transition for non-collegeeducated populations. On the other hand, positive milestones, such as high school graduation or obtaining a first job, which were not represented frequently in the present samples, may assume greater cultural importance and recognition. For individuals who failed to complete high school, noneducational transitional events (e.g., a first job) may be the defining positive episodes of late adolescence. Yet another possibility, counter to Rubin and Berntsen's (2003) original hypothesis, is that predictable negative milestones (e.g., dropping out of school) may appear regularly and prominently both in life scripts and in personal life histories.

Additional research is needed to evaluate more fully Rubin and Berntsen's (2003) conclusion that "cultural knowledge provides powerful constraints for individual memory" (p. 11) or McAdams's (2001) assertion that "life stories mirror the culture wherein the story is made and told" (p. 114). One promising strategy involves identifying variations in cultural expectations for age-related milestones and then searching for corresponding differences in the temporal organization of memories. Cross-cultural studies may be especially informative in this regard (Berntsen \& Rubin, 2004). Conway et al. (2005) found evidence for the reminiscence bump in several different cultures (Japan, China, Bangladesh, England, and the United States), but the extent to which these cultures share a common life script is not entirely clear. Cross-cultural analyses of memory content would indicate whether the bump memories frequently focus on common themes. A stronger test of the life script explanation would involve first identifying positive or negative developmental landmarks that are pervasive only in particular cultures or subcultures and then conducting comparative analyses.

Social scientists have long been interested in possible connections between culture and individual memory. One reflection of this interest is the concept of shared, or collective, memory, which, although widely used, is loosely defined and difficult to subject to strong empirical test (Pillemer, 2004; Wertsch, 2002). The life script hypothesis is especially attractive because it identifies individual memory and culturally prescribed expectations as two separate but interlocking entities. Individuals experience and remember personal events from their own idiosyncratic lives, but the way in which events are encoded and organized in memory is determined, in part, by shared cultural expectations for the typical life course. As such, the concept of life scripts provides a promising tool for examining the interaction of individual memory and collective knowledge. 


\section{AUTHOR NOTE}

Correspondence concerning this article should be addressed to D. B. Pillemer, Department of Psychology, University of New Hampshire, 10 Library Way, Conant Hall, Durham, NH 03824 (e-mail: david .pillemer@unh.edu).

\section{REFERENCES}

Berntsen, D., \& Rubin, D. C. (2002). Emotionally charged autobiographical memories across the life span: The recall of happy, sad, traumatic, and involuntary memories. Psychology \& Aging, 17, 636-652.

Berntsen, D., \& Rubin, D. C. (2004). Cultural life scripts structure recall from autobiographical memory. Memory \& Cognition, 32 , 427-442.

Conway, M. A., \& Pleydell-Pearce, C. W. (2000). The construction of autobiographical memories in the self-memory system. Psychological Review, 107, 261-288.

Conway, M. A., Wang, Q., Hanyu, K., \& Haque, S. (2005). A crosscultural investigation of autobiographical memory: On the universality and cultural variation of the reminiscence bump. Journal of CrossCultural Psychology, 36, 739-749.

Greene, H. R., \& Greene, M. W. (2000). Making it into a top college: 10 steps to gaining admission to selective colleges and universities. New York: Cliff Street Books.

MaYher, B. (1998). The college admissions mystique. New York: Noonday.

McAdams, D. P. (2001). The psychology of life stories. Review of General Psychology, 5, 100-122.

NeIsser, U. (1982). Memory observed: Remembering in natural contexts. San Francisco: Freeman.

PAUL, B. (1995). Getting in: Inside the college admissions process. Reading, MA: Addison-Wesley.

Pillemer, D. B. (1998). Momentous events, vivid memories. Cambridge, MA: Harvard University Press.
Pillemer, D. B. (2004). Can the psychology of memory enrich historical analyses of trauma? History \& Memory, 16, 140-154.

Rosenberg, M. (1965). Society and the adolescent self-image. Princeton, NJ: Princeton University Press.

Ross, M., \& Wilson, A. E. (2002). It feels like yesterday: Self-esteem, valence of personal past experiences, and judgments of subjective distance. Journal of Personality \& Social Psychology, 82, 792-803.

Ross, M., \& Wilson, A. E. (2003). Autobiographical memory and conceptions of self: Getting better all the time. Current Directions in Psychological Science, 12, 66-69.

Rubin, D. C. (1986). Autobiographical memory. Cambridge: Cambridge University Press.

Rubin, D. C. (1996). Remembering our past: Studies in autobiographical memory. Cambridge: Cambridge University Press.

Rubin, D. C., \& Berntsen, D. (2003). Life scripts help to maintain autobiographical memories of highly positive, but not highly negative, events. Memory \& Cognition, 31, 1-14.

Rubin, D. C., Rahhal, T. A., \& Poon, L. W. (1998). Things learned in early adulthood are remembered best. Memory \& Cognition, 26, 3-19.

TeKcan, A. I. (2001). Flashbulb memories for a negative and a positive event: News of Desert Storm and acceptance to college. Psychological Reports, 88, 323-331.

Wertsch, J. V. (2002). Voices of collective remembering. Cambridge: Cambridge University Press.

Wilson, A. E., \& Ross, M. (2001). From chump to champ: People's appraisals of their earlier and present selves. Journal of Personality \& Social Psychology, 80, 572-584.

Wilson, A. E., \& Ross, M. (2003). The identity function of autobiographical memory: Time is on our side. Memory, 11, 137-149.

(Manuscript received July 19, 2005; revision accepted for publication March 1, 2006.) 